\title{
Spectroscopic Characterization of Laser Ablated Germanium Plasma
}

\author{
Muhammad Ashraf ${ }^{1,2 *}$, Nek Muhammad Shaikh², Tasneem Zehra ${ }^{1}$, \\ Ghulam Abbas Kandhro ${ }^{1}$ and Ghulam Murtaza ${ }^{2}$ \\ ${ }^{1}$ Department of Basic Sciences, Mathematics and Humanities, Dawood University of Engineering and Technology, \\ Karachi, Sindh, Pakistan. \\ ${ }^{2}$ Institute of Physics, University of Sindh, Jamshoro, Sindh, Pakistan. \\ *Corresponding AuthorEmail: ashraf7767@yahoo.com \\ Received 12 October 2020, Revised 23 June 2021, Accepted 29 October 2021
}

\begin{abstract}
In the present study, the germanium (Ge) sample has been studied by laser induced breakdown spectroscopy which leads to the formation of plasma plume in the air. This research work comprises on pure Ge sample, and it has been studied using laser irradiance $1.83 \times 10^{11}$ watt. $\mathrm{cm}^{-2}$ and Q-Switched Nd:YAG laser pulse $(\lambda \sim 1064 \mathrm{~nm}$ wavelength and $\tau \sim 5 \mathrm{~ns}$ pulse width). The spatially resolved plasma plume parameters are investigated, such as variation of electron temperature $T_{e}$ and electron number density $n_{e}$ as a function of detector position. These parameters show variation in the plasma plume and yield electron temperature $T_{e}$ from 12340 to $7640 \pm 1200$ $\mathrm{K}$. Whereas electron number density $n_{e}$ varies from $3.6 \times 10^{17}$ to $1.60 \times 10^{17} \mathrm{~cm}^{-3}$ with the change in detector position is moving away from plasma plume from 0 to $3 \mathrm{~mm}$. The results show that electron temperature $T_{e}$ and electron number density $n_{e}$ are estimated from the Boltzmann plot method and by using Lorentzian function at spectral line using FWHM full width at half maximum at $265.11 \mathrm{~nm}\left(4 \mathrm{p} 5 \mathrm{~s}^{3} \mathrm{p}_{2} \rightarrow 4 \mathrm{p}^{2}{ }^{3} \mathrm{p}_{2}\right)$ wavelength of Ge (I) line, respectively.
\end{abstract}

Keywords: LIBS, spectroscopy, germanium, plasma, laser.

\section{Introduction}

In recent years, the application of laser removal of the sample has been widely used for many aspects, included as (analysis techniques, industrial applications, archeological and geological applications) [12]. The technique of laser plasma formation through laser induced breakdown spectroscopy (LIBS) is a complex phenomenon; in this method, the laser is irradiated with material [3-4], and these emissions of radiations are given by optical emission spectroscopy [5-6]. LIBS can be used to analyze any state of matter, either solid, liquid, gas, or aerosols, with the advantages of situ analysis [7-8]. The LIBS is an effective analysis technique and gives the enhanced signal for the analysis of a sample. The ablation method leads to plasma formation and creating high laser irradiance watt. $\mathrm{m}^{-2}$ at the target surface [8-10].

Laser induced plasma is mainly influenced by the pulse duration of laser light, whereas plasma formation time is microseconds [11-15]. In this work, we have used Nd:YAG laser, which has a nanosecond pulse duration. The pulse duration further interacts with plasma plume, which is known as laser plasma interaction [14-15]. Laser plasma interaction gives the absorbance of 
laser light into the plasma plume. The remaining laser light cannot reach the target surface, while some part of laser light is reflected through the surface reflectivity of the sample after plasma formation [16-19].

During the last few years, several research groups have studied various investigations about the analytical performance of Ge plasma [20]. They deposited Ge wafers using laser induced air breakdown technique which formed the layers comprised of nanoscale and Ge nanocrystals [20]. Further groups deposited the Ge films by pulsed laser deposition methods in helium gas atmosphere, and Ge plasma plume was produced by $\mathrm{KrF}$ laser at a laser wavelength of $\lambda=248 \mathrm{~nm}$ [21]. Some more studies have been examined about the temporally and spatially resolved velocity of Ge species by using an excimer laser and producing the $\mathrm{Ge}$ plasma in the oxygen environment [22-24].

\section{Materials and Methods}

LIBS spectrum analysis was performed with the Collection of radiation from plasma plume. It was observed that irradiation of Nd:YAG laser Nova - Quantel, France $(\lambda=1064 \mathrm{~nm}$, with a pulse width of 5 $\mathrm{ns}$, the repetition rate of $10 \mathrm{~Hz}$ ) was used to ablate the plasma plume. LIBS experiment was performed by the duration of $5 \mathrm{~ns}$, and time integration was $3 \mathrm{~ms}$ [25]. The sample was managed in such a way as to prevent air breakdown before the Ge sample surface by using a focusing quartz crystal lens of $20 \mathrm{~cm}$ focal length. The portion of the laser beam irradiated at the sample surface was about 0.5 $\mathrm{mm}$ in diameter. For every laser shot each time, a new surface of Ge sample was provided by $3 \mathrm{~d}$ rotation holder to avoid surface damage caused by high laser irradiance. The LIBS Ocean Optics LIBS2500+ spectrometer system triggered the Q-Switched of the Nd:YAG laser. The
$\mathrm{Nd}$ :YAG laser flash lamp triggered the LIBS 2500+ spectrometer detection system with a synchronized laser pulse. This allowed the spectrum to record in the range from $200 \mathrm{~nm}$ to $800 \mathrm{~nm}$. While processing the digitized light signal into spectrum form, data recorded was an average of 3 laser shots.

\section{Results and Discussion}

Our present research work mentioned the effect of laser produced Ge plasma with variation in distance from plasma plume. This is known to spatially resolve Laser-induced plasma spectroscopy. The above related work is mainly related to the variation in both ionic and atomic transition lines. Moreover, this novel behavior shows the recombination rate in laser ablated Ge plasma; meanwhile, the main objective of our study would help calculate recombination rate, thin films on a substrate, pulse laser deposition, and other plasma plume parameters. This behavior has been influenced by sample property, laser parameters, and ambient conditions on laser ablated Ge.

The plasma plume leads to generate the emission spectrum of a $\mathrm{Ge}$ as given in Fig. 1(a). The emission spectrum of $\mathrm{Ge}$ plasma was at a different distance from the target surface while keeping the other parameters constant. The spectrum region from 200 to $430 \mathrm{~nm}$ is predominantly associated with the neutral Ge lines. As the distance from the Ge plasma increased, the structure of the singly ionized nitrogen seemed to be disappearing. This shows a decrease in relative intensity. Whereas some singly ionized Ge line has also appeared and along with hydrogen alpha line at $656.28 \mathrm{~nm}$ is detected.

Fig. 1(b) shows the observed Ge (I) lines and their associated transitions. This is the enlarged portion of the spectrum that 
covers wavelengths from 240 to $280 \mathrm{~nm}$. All these lines come from the transitions, which are labeled in the spectrum and their corresponding wavelengths according to the LS coupling scheme. The strongest line corresponds to 265.1 and $259.3 \mathrm{~nm}$, and their multiplet triplet structure is assigned as $4 \mathrm{p} 5 \mathrm{~s}$ ${ }^{3} \mathrm{p}_{2} \rightarrow 4 \mathrm{p}^{2}{ }^{3} \mathrm{p}_{1,2}$, whereas weak emission corresponds to 249.8 , and $253.3 \mathrm{~nm}$ and their multiplet structure from singlet to triplet are assigned due to these transition lines $4 \mathrm{p} 5 \mathrm{~s}{ }^{1} \mathrm{p}_{1}$ $\rightarrow 4 \mathrm{p}^{2}{ }^{3} \mathrm{p}_{0,1}$, singlet transition structure of $\mathrm{Ge}$ (I) $4 \mathrm{P} 4 \mathrm{~d}{ }^{1} \mathrm{D}_{2} \rightarrow 4 \mathrm{P}^{2}{ }^{1} \mathrm{D}_{2}$ at $241.73 \mathrm{~nm}$, and triplet transition structure of $\mathrm{Ge}$ (I) $4 \mathrm{P} 5 \mathrm{~s}{ }^{3} \mathrm{P}_{1}$ $\rightarrow 4 \mathrm{P}^{2}{ }^{3} \mathrm{P}_{2}$ at $275.5 \mathrm{~nm}$. Another multiplet triplet structure of Ge (I) $4 \mathrm{P} 5 \mathrm{~s}^{3} \mathrm{p}_{0,1} \rightarrow 4 \mathrm{P}^{2}{ }^{3} \mathrm{P}_{1}$ at 270.9 and $269.1 \mathrm{~nm}$.
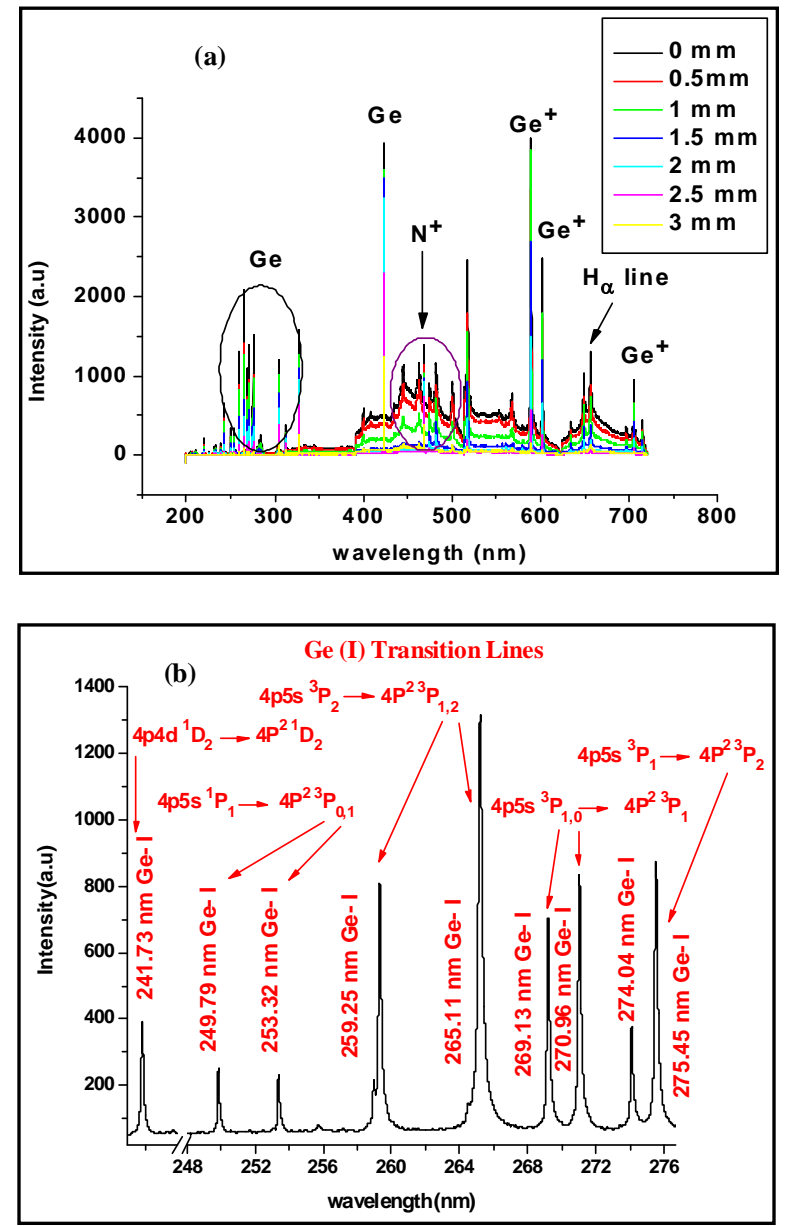

Figure 1. (a) Emission spectrum of germanium plasma generated by $1064 \mathrm{~nm}$ of $\mathrm{Nd}$ :YAG laser pulse at $05 \mathrm{~mm}$ from silicon sample surface at irradiance $1 \times 10^{11} \mathrm{Wcm}^{-2}$, (b) Emission spectrum of germanium plasma predominatel $\mathrm{y}$ associated with Ge Ilines
Besides the $\mathrm{Ge}$ (I) lines, we also observed Ge (II) lines, the strong emission at 589.3 and $602.1 \mathrm{~nm}$. A doublet due to the $4 \mathrm{~s}^{2} 5 \mathrm{p}^{2} \mathrm{p}_{3 / 2,1 / 2} \rightarrow 4 \mathrm{~s}^{2} 5 \mathrm{~s}^{2} \mathrm{~S}_{1 / 2}$ transition of $\mathrm{Ge}$ (II) singly ionized spectral line. In the following section, we will discuss the plasma parameters those has been analyzed from the emission spectrum of laser produce $\mathrm{Ge}$ plasma.

In the present study with the LS coupling scheme, it was observed that Ge neutral transition lines through LS coupling rules. From these rules, the ground state configuration comes to be $4 s^{2} 4 p^{2}$, which has five sub levels, namely ${ }^{3} \mathrm{P}_{0}{ }^{3} \mathrm{P}_{1}{ }^{3} \mathrm{P}_{2}{ }^{1} \mathrm{D}_{2}{ }^{1} \mathrm{~S}_{0}$. These sub levels were drawn in increasing energy levels, and their associated excited states are ${ }^{3} \mathrm{P}_{0}{ }^{3} \mathrm{P}_{1}{ }^{3} \mathrm{P}_{2}{ }^{1} \mathrm{P}_{1}$, and these excited states are attached with the state $4 \mathrm{p} 5 \mathrm{~s}$. This has four sub levels, whereas, for the $4 \mathrm{p} 4 \mathrm{~d}$ excited state, there exist 7 sub levels ${ }^{1} \mathrm{D}_{2}{ }^{3} \mathrm{D}_{2}{ }^{3} \mathrm{D}_{1}{ }^{3} \mathrm{D}_{3}{ }^{3} \mathrm{~F}_{2}{ }^{3} \mathrm{P}_{2}{ }^{1} \mathrm{~F}_{3}$, all the excited states, and their ground states are illustrated in Fig. 2.

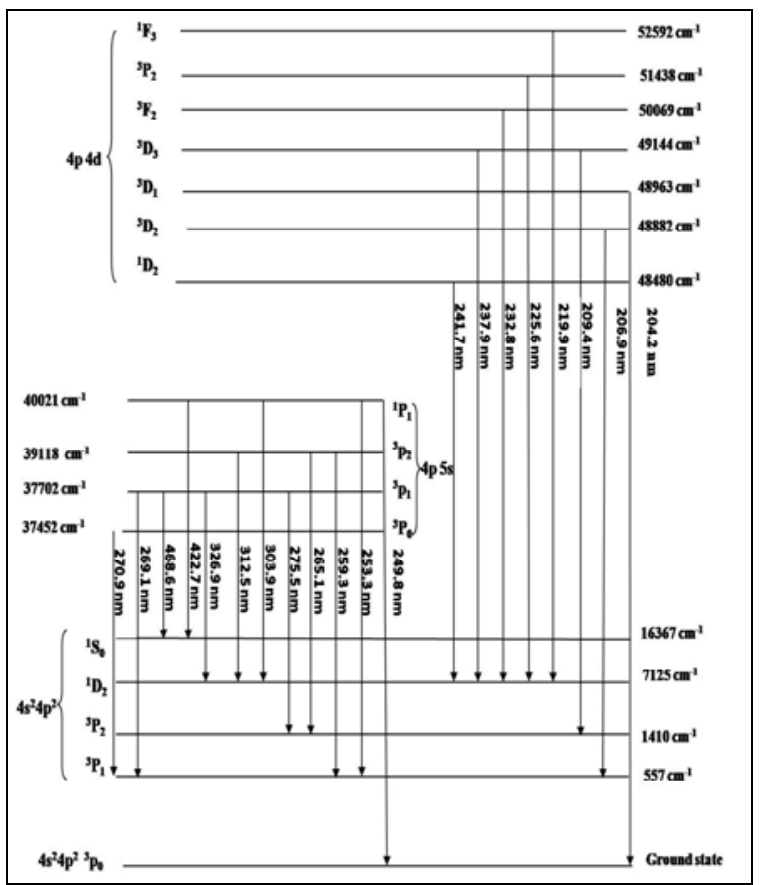

Figure 2. Energy level diagram of Ge - I lines observed in present research work 
Recombination Rate in Germanium at different distances. It was found that Transition Lines

according to the LS coupling scheme, the strongest line corresponds to Ge (I) $265.1 \mathrm{~nm}$

Current work also comprises the and $\mathrm{Ge}(\mathrm{I}) 259.2 \mathrm{~nm}$, and their multiplet triplet variation in the intensity of Ge transition lines structure is assigned as $\left(4 \mathrm{p} 5 \mathrm{~s}^{3} \mathrm{p}_{2} \rightarrow 4 \mathrm{p}^{2}{ }^{3} \mathrm{p}_{1,2}\right)$.

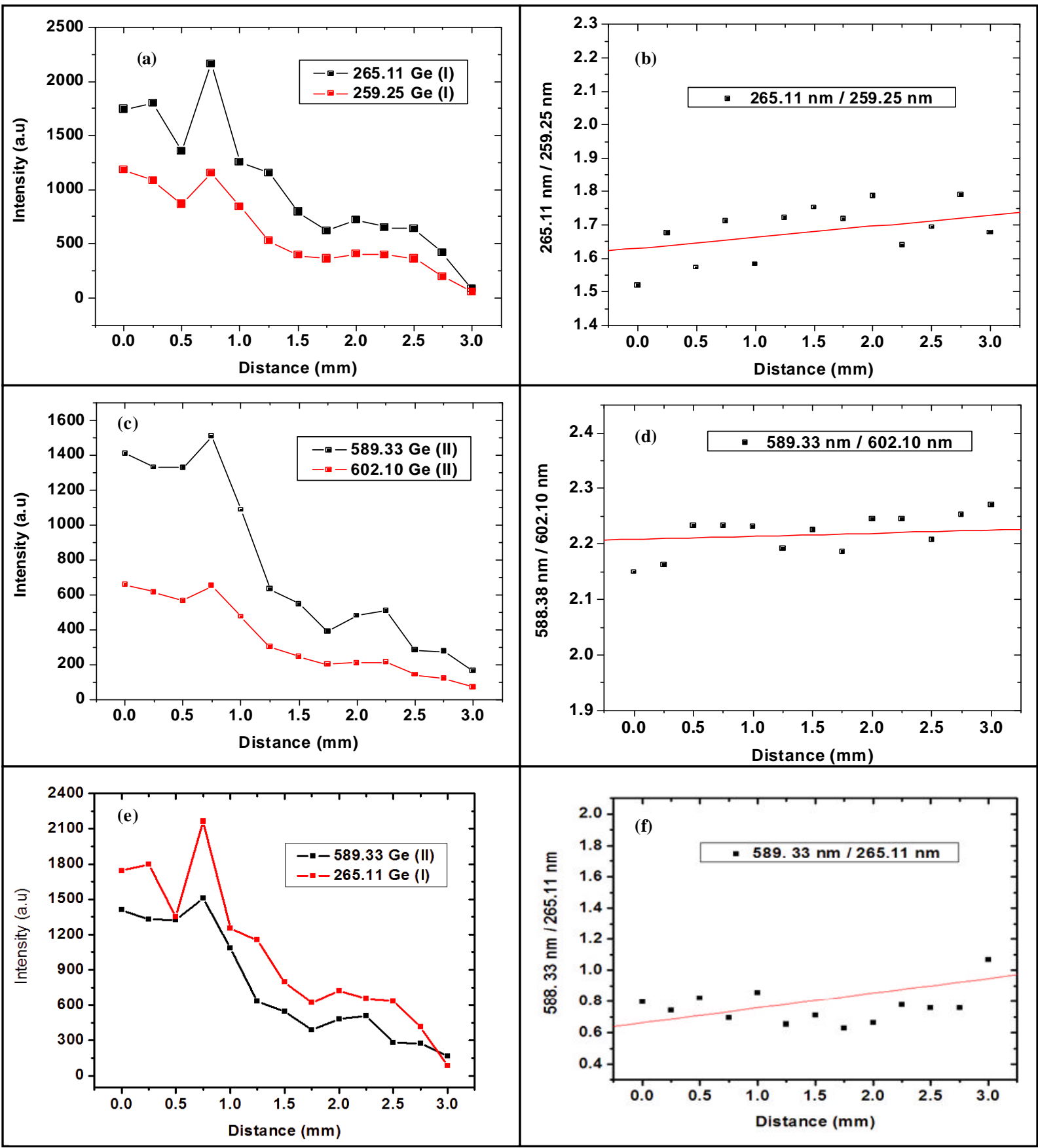

Figure 3(a), (b) represent variation in intensity and their ratio at Ge $\mathbf{I} 265.11 \mathrm{~nm}$ and $259.25 \mathrm{~nm}$, (c), (d) represents variation in intensity and their ratio at Ge II $589.33 \mathrm{~nm}$ and $602.10 \mathrm{~nm}$, (e), (f) represent variation in intensity at Ge I $265.11 \mathrm{~nm}$ and $\mathrm{Ge}$ II $589.33 \mathrm{~nm}$ 
We also observed that Ge (II) lines the strong emission at $589.3 \mathrm{~nm}$ and $602.1 \mathrm{~nm}$ a multiplet doublet due to the $\left(4 \mathrm{~s}^{2} 5 \mathrm{p}^{2} \mathrm{p}_{3 / 2,1 / 2} \rightarrow\right.$ $4 s^{2} 5 s^{2} S_{1 / 2}$ ) transition of Ge (II) singly ionized spectral line. Fig. 3(a) and Fig. 3(b) represent variation in intensity and the intensity ratio of triplet at $265.1 \mathrm{~nm}\left(4 \mathrm{p} 5 \mathrm{~s}^{3} \mathrm{p}_{2} \rightarrow 4 \mathrm{p}^{2}{ }^{3} \mathrm{p}_{1}\right), 259.2$ $\mathrm{nm}\left(4 \mathrm{p} 5 \mathrm{~s}^{3} \mathrm{p}_{2} \rightarrow 4 \mathrm{p}^{2}{ }^{3} \mathrm{p}_{2}\right)$. Similar pattern of variation in intensity and ratio of doublet to doublet structure transition of $\mathrm{Ge}$ ionic (II) lines at $589.3 \mathrm{~nm}\left(4 \mathrm{~s}^{2} 5 \mathrm{p}^{2} \mathrm{p}_{3 / 2} \rightarrow 4 \mathrm{~s}^{2} 5 \mathrm{~s}^{2} \mathrm{~S}_{1 / 2}\right)$ and $602.1 \mathrm{~nm}\left(4 \mathrm{~s}^{2} 5 \mathrm{p}^{2} \mathrm{p}_{1 / 2} \rightarrow 4 \mathrm{~s}^{2} 5 \mathrm{~s}^{2} \mathrm{~S}_{1 / 2}\right)$ is shown in Fig. 3(c) and Fig. 3(d), respectively. Another study of Ge neutral transition Ge (I) with ionic transition Ge (II) triplet and doublet structure from Fig. 3(e) and Fig 3(f). Which represents variation in intensity and also the intensity ratio at $265.1 \mathrm{~nm}\left(3 \mathrm{p} 4 \mathrm{~s}{ }^{1} \mathrm{p}_{1} \rightarrow 3 \mathrm{p}^{2}\right.$ $\left.{ }^{1} \mathrm{D}_{2}\right)$ and $589.3 \mathrm{~nm}\left(3 \mathrm{~s}^{2} 4 \mathrm{p}^{2} \mathrm{p}_{3 / 2} \rightarrow 3 \mathrm{~s}^{2} 4 \mathrm{~s}^{2} \mathrm{~S}_{1 / 2}\right)$. Hegazy et al. [26] worked on the ratios of several transitions at the wavelength ratios of coppers neutrals 324.7 / $327.2 \mathrm{~nm}$ and on manganese neutrals 478.3 / $475.4 \mathrm{~nm}$ further on magnesium neutrals $518.4 \mathrm{~nm} / 517.3 \mathrm{~nm}$. Hegazy et al. [27] same authors group, further worked on ratios of several transitions at wavelength ratios of tin ionic $325.3 \mathrm{~nm} \mathrm{/}$ $321.2 \mathrm{~nm}$. Santos et al. [23] worked on ratios of several transitions of $\mathrm{Ge}^{3+} / \mathrm{Ge}^{+}, \mathrm{Ge}^{3+} / \mathrm{Ge}$, $\mathrm{Ge}^{+} / \mathrm{Ge}$ using the different laser irradiance. In the present research work, the intensity variation and their ratios are followed by the relation.

$$
I(i j)=\frac{h v}{4 \pi} N_{i} g_{i} A_{i j}
$$

The comparison of Fig. 3(a) with Fig.3(c) reveals that the intensity of $\mathrm{Ge}$ neutrals in Fig. 3(a) decays exponentially. Whereas the Ge ionic lines in Fig. 3(c) follow different decay rates, Fig. 3(e) represents another study for $\mathrm{Ge}$ ionic with neutral. Besides, it is also deduced from the comparison of Fig. 3(d) and Fig. 3(f) the intensity ratio of $\mathrm{Ge}$ ionic lines and $\mathrm{Ge}$ ionic with neutral is low with Fig. 3(b), which is the intensity ratio of Ge neutral lines. The above study of ionic species shows a different trend with the neutral species of $\mathrm{Ge}$ plasma. Considering this work, this seems that the distance variation parameter predominantly affects ionic lines and also neutral lines. This happens with the change in distance from detector position to spectrometer.

\section{Te and Ne Variation in Germanium Plasma Plume}

The present research work electron temperature of Ge plasma has been estimated by using the Boltzmann plot method [25, 28]. The electron temperature of Ge plasma is an important parameter that comes from the technique of laser induced emission spectroscopy employed to generate the plasma plume.

$$
\ln \left(\frac{\lambda_{i j} I_{i j}}{h c A_{i j} g_{i j}}\right)=\ln \left(\frac{N(T)}{z}\right)-\frac{E_{i}}{K T}
$$

When left hand side of eq (2) is plotted vs upper level energy. This yields the electron temperature of Ge plasma through Boltzmann plot by using five Ge neutrals transitions lines, which are included as, 269.1, 241.7, 259.6, 270.9 , and $275.5 \mathrm{~nm}$. These lines are free from self absorption and assumed from the peak profile shape, and no variation was observed except intensity variation in these spectral lines. NIST database was used to calculate plasma parameters [29], as given in Table 1 . The estimated electron temperature of $\mathrm{Ge}$ plasma is found to be 12340 to $7640 \mathrm{~K}$ with the spatially resolved manner from $\mathrm{Ge}$ sample surface with systematic error is 12340 $\pm 1200 \mathrm{~K}$. This rises from the calculated intensity of peaks used and transition probability of lines. 
Table 1. Spectroscopic data for germanium spectral lines.

\begin{tabular}{cccc}
\hline Wavelength $(\boldsymbol{n m})$ & $\boldsymbol{g}_{\boldsymbol{k}}$ & $\boldsymbol{A}_{\boldsymbol{i k}}\left(\boldsymbol{s}^{-1}\right)$ & $\boldsymbol{E}_{\boldsymbol{k}}\left(\boldsymbol{c m}^{-1}\right)$ \\
\hline 241.7 & 5 & $9.6 \mathrm{E}+07$ & 48480 \\
259.3 & 5 & $7.1 \mathrm{E}+07$ & 39118 \\
270.9 & 1 & $2.8 \mathrm{E}+08$ & 37452 \\
275.5 & 3 & $1.1 \mathrm{E}+08$ & 37702 \\
269.1 & 3 & $1.8 \mathrm{E}+08$ & 37702 \\
\hline
\end{tabular}

The detailed information about electron temperature for Ge plasma observed at $0.3 \mathrm{~mm}$ distance was $12340 \mathrm{~K}$. The electron temperature of Ge plasma is $7640 \mathrm{~K}$, and again with the increase in the distance at 0.9 $\mathrm{mm}$ apart same decreasing trend was observed, at $1 \mathrm{~mm}$ distance the electron temperature difference start increases till 3 $\mathrm{mm}$ far from plasma creation point. The result obtained that the order of decreasing factor for electron temperature is 1.61. The plasma recombination rate shows that as the distance from the plasma creation point increases, its recombination rate decreases [30-31].

Plasma parameters are essential specifications for the generation of nanoparticles and thin films on the substrate. For these reasons, electron number density is essential. Moreover, electron number density has been estimated using Ge transition line at wavelength $265.1 \mathrm{~nm}\left(4 \mathrm{p} 5 \mathrm{~s}{ }^{3} \mathrm{p}_{2} \rightarrow 4 \mathrm{p}^{2}{ }^{3} \mathrm{p}_{2}\right)$ and taking the stark width broadening by using the Lorentzian function. The same method has been employed by diverse groups [32-33], as given in Eq. 3.

$$
N_{e} \approx\left(\frac{\Delta \lambda_{1 / 2 F W H M}}{2 w}\right) 10^{16}
$$

In order to calculate electron number density, Lorentzian fitting was employed, and remaining features such as $w$ values were taken from reference data. This $w$ value is known to be the electron impact width parameter. Using these parameters, calculated electron number density for Ge plasma lies in the range of $3.64 \times 10^{17} \mathrm{~cm}^{-3}$.
The laser produce plasma parameters can be counted from LTE plasma. These parameters, electron number density, electron temperature, are followed by electron collision. This creates a high electron number density, and as a consequence, a high collision rate is resulted [34]. At $265.11 \mathrm{~nm}$ wavelength, this transition should be free from self absorption. This self absorption phenomenon was counted from the method of peak profile, and no variation in the shape of $265.11 \mathrm{~nm}$ wavelength was observed at spatial and irradiance parameters.

\section{Conclusion}

The spectroscopic study from $\mathrm{Ge}$ plasma was investigated at variation in distance from detector position to sample surface. Plasma parameters such as electron temperature and electron number density were verified by LTE plasma. Distance variation parameter, known to be spatially resolved, strongly influences electron temperature and electron number density of $\mathrm{Ge}$ plasma. Ge sample surface, where plasma created, showed a high decreasing trend in spectral lines, whereas at far from $\mathrm{Ge}$ sample surface, it showed decreasing trend in spectral lines. Moreover, ionic lines decayed rapidly than neutral lines. The spectroscopic studies of these transition lines at spatially resolved regimes revealed that the decreasing factor for Ge neutrals at 265.1, 259.3, was 30.75, and 20.73 , respectively, and the decrease in ratio $265.1 / 259.3 \mathrm{~nm}$ was 1.0. The decreasing factor for Ge ionic lines 589.3 and $602.1 \mathrm{~nm}$ were 8.54 and 9.37 , respectively, and the decrease in the ratio of ionic line 589.3/602.1 nm was 0.91 .

\section{Conflict of interest}

The authors declare no conflict of interest. 


\section{References}

1. A. A. llyin and S. S. Golik, Spectrochim. Acta B, 87 (2013) 192. doi.org/10.1016/j.sab.2013.06.001.

2. A. A. Bolshakov, J. H. Yoo, C. Liu, J. R. Plumer and R. E. Russo, Appl. Opt., 49 (2010) C132.

doi.org/10.1364/AO.49.00C132.

3. A. Giakoumai, K. Melessanaki and D. Anglos, Anal. Bioanal. Chem., 387 (2007) 749. doi.org/10.1007/s00216-006-0908-1

4. S. Qiao, Y. Ding, D. Tian, L. Yao and G. Yang, Appl. Spectrosc. Rev., 50 (2015) 1.

doi.org/10.1080/05704928.2014.911746.

5. L. B. Guo, B. Y. Zhang, X. N. He, C. M. Li, Y. S. Zhou, T. Wu, J. B. Park, X. Y. Zheng and Y. F. Lu, Opt. Express, 20 (2012) 1436. doi.org/10.1364/OE. 20.001436.

6. M. Cvejić, J. Phys. Conf. Ser., 56 (2014) 5012014.

https://iopscience.iop.org/article/10.1088 /1742-6596/565/1/012014.

7. M. A. Khater, Spectrochim. Acta B, 81 (2013) 1 .

doi.org/10.1016/j.sab.2012.12.010.

8. M. E. Asgill and D. W. Hahn, Spectrochim. Acta B, 64 (2009) 1153. doi.org/10.1016/j.sab.2009.07.026.

9. L. Liu, S. Li, X. N. He, X. Huang, C. F. Zhang, L. S. Fan, M. X. Wang, Y. S. Zhou, K. Chen, L. Jiang, J. F. Silvain and Y. F. Lu, Opt. Express, 22 (2014) 7686.

doi.org/10.1364/OE.22.007686.

10. C. Lopez-Moreno, K. AmponashManager, B. W. Smith, I. B. Gornushkin, N. Omenetto, S. Palanco, J. J. Laserna and J. D. Winefordner, $J$. Anal. At. Spectrom., 20 (2005) 552. doi.org/10.1039/B419173K.

11. A. J. Ball, V. Hohreiter and D. W. Hahn, J. Appl. Spectrom., 59 (2005) 348. doi.org/10.1366/0003702053585282.

12. M. Muller, I. B. Gornushkin, S. Florek and D. Mory, Anal. Chem., 79 (2007) 4419. doi.org/10.1021/ac0621470.

13. I. B. Gornushkin, S. V. Shabanov, N. Omenetto and J. D. Winefordner, $J$. Appl. Phys., 100 (2006) 1. doi.org/10.1063/1.2345460.

14. H. Hou, Y. Li, Y. Tian, Z. Yu and R. Zheng, J. Appl. Phys. Express, 7 (2014) 032402 . doi.org/10.7567/APEX.7.032402.

15. A. N. Kadachi and M. A. Al-Eshaikh, Spectrosc. Lett., 48 (2015) 403. doi.org/10.1080/00387010.2014.895387.

16. Ph. Rohwetter, J. Yu, G. Méjean, K. Stelmaszczyk, E. Salmon, J. Kasparian, J.-P. Wolf and L. Woste, J. Anal. At. Spectrom., 19 (2004) 437.

doi.org/10.1039/B316343A.

17. D. H. Lee, T. H. Kim, E. C. Jung and J. Yun, J. Appl. Phys., 104 (2011) 863. doi.org/10.1007/s00339-011-6427-8.

18. X. Bai, Q. Ma, M. Perrier, V. MottoRos, D. Sabourdy, L. Nguyen, A. Jalocha and J. Yu, Spectrochim. Acta B, 87 (2013) 27. doi.org/10.1016/j.sab.2013.05.019.

19. M. Hanif, M. Salik and M. A. Baig, Opt. Spectrosc., 116 (2014) 315. doi.org/10.1134/S0030400X1402009X.

20. A. V. Kabashin, F. Magny and M. Meunier, J. Appl. Phys., 101 (2007) 054311. doi.org/10.1063/1.2435801.

21. D. Riabinina, M. Chaker and F. Rosei, $J$. Appl. Phys., 89 (2006) 131501. doi.org/10.1063/1.2356689.

22. F. Vega, C. N. Afonso and J. Solis, J. Appl. Phys., 73 (1993) 2472. doi.org/10.1063/1.353106.

23. M. Santos, L. Díaz, J. J. Camacho, J. M. L. Poyato, J. Pola and T. Krenek, Appl. Phys. A, 99 (2010) 811. doi.org/10.1007/s00339-010-5592-5. 
24. M. P. Chuchman and A. K. Shuaibov, Plasma Phys. Rep., 34 (2008) 306. doi.org/10.1134/S1063780X08040077.

25. G. Murtaza, N. M. Shaikh, G. A. Kandhro and M. Ashraf, Spectrochim. Acta A, 223 (2019) 117374. doi.org/10.1016/j.saa.2019.117374.

26. H. Hegazy, E. A. Abdel-Wahab, F. M. Abdel-Rahim, S. H. Allam and A. M. A. Nossair, Arab J. Nucl. Sci. Appl., 47 (2014) 80.

http://www.esnsaeg.com/download/resea rchFiles/(1).pdf.

27. H. Hegazy, H. A. Abd El-Ghany, S. H. Allam and T. M. El-Sherbini, Appl. Phys. B, 110 (2013) 509. doi.org/10.1007/s00340-012-5287-z.

28. J. Iqbal, R. Ahmed, M. Anwar-ul- Haq and M. A. Baig, Laser Phys., 26 (2016) 076001. doi.org/10.1088/1054660X/26/7/076001.
29. NIST Atomic Spectra Database, http://physics.nist.gov/PhysRefData/AS D/lines form.html.

30. H. Shakeel, S. Arshad, S. U. Haq and A. Nadeem, Phys. Plasma, 23 (2016) 053504. doi.org/10.1063/1.4948620.

31. J. Iqbal, R Ahmed and M. A. Baig, Laser Phys., 27 (2017) 046101. doi.org/10.1088/1555-6611/aa5cec.

32. M. A. Baig, M. A. Fareed, B. Rashid and R. Ali, Phys. Plasma, 18 (2011) 083303. doi.org/10.1063/1.3625552.

33. A. Lesage, AIP Conf. Proc., 1058 (2008) 357. doi.org/10.1063/1.3026480.

34. N. M. Shaikh, S. Hafeez and M. A. Baig, Spectrochim. Acta B, 62 (2007) 1311. doi.org/10.1016/j.sab.2007.10.028. 